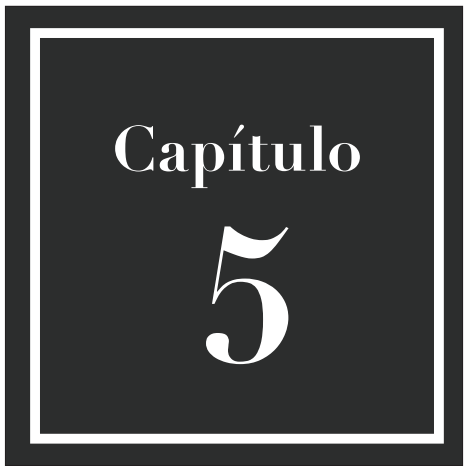

\title{
MANEJO Y ACONDICIONAMIENTO DE LOS HUMEDALES URBANOS COMO SOLUCIÓN A LA PROBLEMÁTICA AMBIENTAL
}

Luego de tratar los diferentes aspectos en capítulos anteriores, como antesala para lograr la administración de los espacios de los humedales urbanos, se debe seleccionar una serie de estrategias orientadas a integrar la conservación y el uso racional de estos ecosistemas, con el fin de generar la simbioempatía como eje de articulación hacia una responsabilidad social compartida hombre-naturaleza. Estas estrategias deben dirigirse a la promoción de la educación ambiental que conllevan a una condición comprometida con respecto al uso racional de los recursos y, por lo tanto, a un desarrollo sostenible. Si bien los procesos de capacitación contribuyen al conocimiento en pro de la conservación y manejo sustentable de los recursos naturales, es entendible que para lograr cambios en la comunidad es preciso desarrollar trabajos que conlleven a un esmero intensivo de la educación ambiental en todos los procesos de capacitación, de tal manera que las soluciones se basen en tácticas exhaustivas donde los diferentes sectores de la sociedad desempeñen un rol trascendental en la solución de las dificultades.

Por tanto, tiene mayor importancia a nivel comunitario, pues la capacitación está diseñada bajo el enfoque pedagógico de "aprender haciendo" al enfrentar a la comunidad a los problemas de la realidad derivados de la experiencia bajo las corrientes holísticas y de la sostenibilidad/sustentabilidad, a su vez que está dirigida a la adquisición de conocimientos ecológicos en relación con estos espacios y a un conocimiento crítico, desde el análisis de los procesos socioambientales y sus consecuencias, desarrollando actitudes y comportamientos vinculados con la conducta que solicita el orden social.

El fin primordial es aplicar estrategias de educación ambiental dirigidas a la comunidad, habitante y aledaña, a fin de que conozca y ponga en práctica el manejo sustentable de los humedales urbanos, llevando a cabo el diagnóstico del área desde el punto de vista físico natural y socioeconómico para determinar conocimientos y necesidades de la población sobre la conducción de los ecosistemas; de esta manera, por medio de un material didáctico tendiente a sensibilizar, conocer y manejar los recursos naturales y cumplir con la ejecución un programa de acción social para la comunidad sobre el manejo sustentable de este territorio.

El procedimiento para lograr un cambio de actitud en una comunidad en relación con un 
problema socio ambiental constituye un reto, puesto que involucra una serie de factores o aspectos intra y multidisciplinarios, que está referido al conocimiento de las necesidades de formación de la comunidad, ejecución del programa educativo ambiental, que incluya actividades educativas, diagramación e ilustración de los materiales instruccionales, talleres, y realización de los talleres para la capacitación a la comunidad.

Antes de continuar se hace necesario revisar el soporte epistemológico o bases teóricas principales, que sirven de pilar cognitivo del plan de acción, en este caso de la estrategia escogida para su ejecución. Estos son los siguientes: manejo, definido como el conjunto detallado de actividades que, producto de una evaluación ambiental, están orientadas a prevenir, mitigar, corregir o compensar los impactos y efectos ambientales que se causen por el desarrollo de un proyecto, obra o actividad (Alcaldía local de Tunjuelito, 2009).

La educación ambiental, según expresó Rengifo (2012), "es un proceso que reconoce valores y aclara conceptos centrados en fomentar las actitudes, destrezas, habilidades y aptitudes necesarias para comprender y apreciar las interrelaciones entre el ser humano, su cultura y la interrelación con la naturaleza" (p.6). De acuerdo con esto, la educación es primordial para adquirir conciencia, valores, técnicas y comportamientos ecológicos y éticos en armonía con el desarrollo sostenible y que favorezcan la participación comunitaria segura en decisiones.

Por su parte, la agricultura urbana se refiere a las técnicas y conocimientos para cultivar la tierra y la producción de alimentos en áreas urbanizadas. Es considerado como el sector económico más amplio del mundo y el número de personas involucradas en él son más numerosas que en todas las otras ocupaciones juntas. Se desarrolla dentro de los límites de las ciudades de todo el mundo e incluye los productos de las actividades agropecuarias, pesqueras y forestales, así como los servicios ecológicos que proporcionan (Navarro, 2013). Y el intercambio de saberes, como el aprendizaje mutuo entre la comunidad mediante el diálogo, exponiendo sus experiencias entre cada integrante (Goyo, 2013).

Al aclarar estos términos, seguidamente se presenta el plan de capacitación, como parte del manejo, que tiene como fin la consecución de su preservación al promover la cultura conservacionista a manera de concienciar a la comunidad, mediante la aplicación de medidas educativas correctivas que garanticen su permanencia en el tiempo como ente fundamental de recreación y hasta como herramienta de investigación y potencial ecoturístico para la región, aparte de todas las bondades propias de los mencionados ecosistemas. Por lo planteado, es necesario el cumplimiento de metas englobadas en los objetivos que conllevan al manejo de los humedales en términos de recursos y esfuerzos, que expresan las acciones y las decisiones que se deben tomar para solucionar los problemas priorizados. La comunidad aledaña a los humedales es la principal actora en la ejecución de las actividades.

Por otra parte, instaurar las medidas necesarias enfocadas al manejo y gestión integral de los humedales urbanos para la recuperación, restauración y conservación, acordes con sus particularidades actuales y potenciales, como resultado de un proceso de planificación participativa, que permitan el mantenimiento de los servicios ambientales y atributos biológicos, ecológicos y culturales de este ecosistema. Este planteamiento se refiere a:

- Recuperar el inmenso potencial ecológico de los humedales e integrarlos al paisaje urbano.

- Crear trabajos de conservación, protección y uso sostenible de los humedales, mediante componentes que aseguren su permanencia en el tiempo.

- Transformar la oferta de hábitats, beneficiando el establecimiento y sostenimiento de especies presentes y otras desaparecidas en los humedales.

- Fortalecer las áreas para la gestión ambiental participativa, educación, investigación y recreación pasiva dentro de los humedales.

- Fundar espacios de participación ciudadana en la gestión, medición y sostenibilidad de los humedales a largo plazo, que conduzcan a la privación social. 
- Perfeccionar el medio urbanístico del área de influencia.

A partir de allí, la promoción del comportamiento solidario, a la vez que contempla formas participativas de planificación del territorio, trata de conformar áreas de acción concertada para una gestión más apropiada. Su implementación requiere el uso de técnicas que permitan la concreción de estudios básicos, detallados y continuos: hidráulicos, de suelo y urbano; simultáneamente, la evaluación ecológica de la importancia, estado y funcionamiento del humedal, las posibilidades de recuperación, remediación, conservación y mantenimiento, el inventario sobre la situación ambiental y el fortalecimiento, revisión y actualización de la información existente y completamiento y actualización de la base de datos de estudios teóricos y de campo creada.

Completar y poner en vigencia los estudios de los humedales y realizar estudios de detalle de toda la cuenca hidrográfica de aporte al mismo y del sistema de desagües pluviales existentes, especialmente su grado de contaminación.

La materialización en el terreno, que separa legalmente el ámbito público del privado, paso previo a la implementación de cualquier estrategia de organización del uso del suelo y del agua, superando los enfoques fragmentados que también se manifiestan respecto a las responsabilidades de manejo de cada cuenca.

Se debe destacar que para el manejo integrado de los humedales urbanos, tiene que ser respaldado por leyes e instrumentos de gestión local adecuados, incluso los instrumentos económicos que hagan posible y faciliten su planificación, armonizando con legislaciones y políticas local, nacional e internacional, al igual que elaborar normativas que faciliten la aplicación de las nuevas políticas, creando comités de cuencas, introduciendo incentivos económicos, penalidades y reglamentando las actividades susceptibles de impactar negativamente en el manejo de los humedales urbanos.

La introducción de cambios en la estructura administrativa en forma gradual y permanente se refiere a:

a. Participación y asistencia entre organismos competentes del manejo de los recursos naturales y urbanos, la protección del ambiente y el manejo del suelo urbano con el humedal contenidos en él y también con las universidades, entre otros organismos.

b. Autoridad coordinadora con representantes de estos organismos, responsable de programas eficaces de administración del suelo y el ambiente, que promueva acciones gubernamentales conjuntas y monitoree los programas susceptibles de impactar en la cuenca y su humedal.

c. Revisión y actualización periódica de las normas en virtud del desarrollo que vayan sufriendo los sitios o ante la demanda de nuevos emprendimientos que impacten sobre el sector.

Desarrollar programas de mejoramiento ambiental, con la activa gestión de los vecinos implicados y la supervisión del gobierno local en todas sus instancias, donde se deberán encarar acciones de revitalización urbana y de restauración de los humedales, en función de las prioridades y fondos disponibles, contemplando los siguientes aspectos: restauración de los humedales, materialización del "bosque protector" y espacios recreativos en los humedales, programa de ornato en la zona urbanizada: plantación y mantenimiento, programa de higiene urbana, con especial énfasis en la problemática de los residuos sólidos domiciliarios, su reciclaje, reducción y adecuada disposición, formación de grupos de guardería ambiental y ejecución de obras básicas de infraestructuras y equipamientos urbanos, entre otros.

En otro orden de ideas, y considerando que los problemas ambientales no se resuelven solamente a partir la educación, se plantea la necesidad de abordar la solución desde un marco más amplio: la gestión ambiental donde la educación es un eslabón más de la gestión. Puesto que el hecho informativo o de divulgación sirve de disparador al proceso de conocimiento o de contenidos referidos a las conductas y actitudes positivas, individuales y grupales, consideradas moral y socialmente 
relevantes como son las normativas, los hábitos de buen uso de un equipamiento, las conductas frente a las otras especies, en especial árboles y aves, los hábitos de higiene, el correcto manejo de los residuos domiciliarios, el conocimiento de las funciones de los humedales y su interdependencia con el entorno, entre otros.

Haciendo hincapié en la participación ciudadana que es básica, sin ella es impensable la aplicación y sustentabilidad de cualquier medida que se promueva para la preservación y/o restauración de los humedales. La Municipalidad, como instancia gubernamental más próxima a la gente, adquiere un rol importante en cuanto a su posibilidad de amplia convocatoria a los actores sociales afectados. He aquí la importancia de la educación ambiental, presentando algunos temas a desarrollar dentro del programa básico:

- El ambiente urbano y la identidad.

- Análisis del contexto.

- Reconocimiento y valoración de los humedales. Su problemática y potencialidades.

- Interrelación de los componentes ambientales.

- La gestión comunitaria y los espacios de uso público.

- La salud humana y la contaminación ambiental.

- Rescate de los espacios degradados y los espejos de agua.

- Manejo de los residuos sólidos urbanos.

- Reforestación y ornato urbano y predial. Mantenimiento y reposición.

- El desarrollo sustentable y la actividad productiva.

- Propuestas alternativas, individuales y grupales, de solución a las situaciones encontradas.
En cuanto a la evaluación y monitoreo ambiental, y con el fin de representar ciertos aspectos del estado del ambiente, de la disponibilidad de recursos naturales y de las actividades humanas relacionadas con ellos, resulta conveniente contar con indicadores de sustentabilidad que se constituyan en información ambientalmente creíble, tendiente a facilitar la formación de opinión para la toma de decisiones, públicas o privadas. Se recomienda desarrollar los indicadores básicos:

- Indicadores de cambio del ambiente, característicos de la disposición del medio y de la calidad de los recursos naturales asociados a procesos de explotación socioeconómica. Se parte de la situación detectada al momento de poner en funcionamiento este mecanismo de evaluación de resultados

- Indicadores de respuesta, indicativos del atrevimiento social y político en su componente ambiental y de recursos naturales. Se irán registrando con la periodicidad que cada uno de los temas requiera.

Los posibles indicadores básicos serían el nivel de vulnerabilidad ambiental, en especial al riesgo hídrico; concentración demográfica y situaciones de tenencia irregular de la tierra; deterioro de la calidad de vida; eliminación de residuos y contaminación por vertidos de aguas residuales; reingreso de aves y peces al humedal, entre otros.

De acuerdo con la transferencia de resultados debe efectuarse a diferentes niveles y complejidades de comprensión y utilización:

a. Instituciones públicas y privadas vinculadas al manejo de los recursos hídricos, del ambiente, las infraestructuras y servicios públicos, educación y salud, la planificación urbana y vivienda, entre otros.

a. Instituciones académicas y científicas. Profesionales y técnicos. Las entidades pro- 
fesionales y de investigación podrán contribuir con la formación de recursos humanos y profesionales comprometidos con la multidisciplinariedad en el estudio del ambiente y su planificación con criterios de desarrollo sustentable.

a. La comunidad en general, en la que mediante la transferencia se pretende contribuir a formar una conciencia ambiental, incentivando el sentido de su participación y de la solidaridad en la búsqueda y solución integral de los problemas de cada microcuenca y del conjunto. Los medios de comunicación brindan un apoyo significativo a la necesaria transferencia, dada su amplia difusión masiva.

Una vez alcanzada la reconstrucción o recuperación para toda el área de los humedales se realiza la zonificación con los siguientes fines: mejoramiento de la calidad del agua, recuperación de hábitats terrestres y acuáticos, preservación y protección ambiental y de educación y recreación pasiva. A continuación se desglosa cada una de ellas.

\section{ZONA PARA LA MEJORA DE LA GALIDAD DEL AGUA}

El principal uso en ella será la implementación de sistemas físicos y biológicos de tratamiento de los afluentes de los humedales, mediante procesos sencillos de separación de residuos sólidos y depuración de aguas con vegetación macrófita acuática.

Uso compatible: utilización de la zona como hábitat de alimentación y anidación de fauna.

Uso condicionado: la zona también puede ser usada como sitio de investigación, con los debidos permisos y seguimiento.

Uso prohibido: ingreso y tránsito del público, puesto que claramente significaría riesgos para la salud y seguridad de la población.

\section{ZONA DE REGUPERAGIÓN DE HÁBITATS TERRESTRES Y AGUÁTICOS}

Los diferentes hábitats propuestos dentro de esta zona son:

Terrestres: corresponden a unas delgadas franjas entre el cuerpo de los humedales como mecanismo de protección contra predadores y refugio de algunas especies de fauna; además, permite aislar al humedal de los diferentes disturbios antrópicos generados por la presión ejercida por el área contigua a cada humedal.

Bosque protector: el objetivo es generar refugio y alimentación para fauna, la cual estará integrada por árboles de crecimiento rápido y con follaje denso que atraigan fauna y produzcan sombra para ayudar a controlar la maleza, además de crear condiciones de un bosque de cercanías a cada humedal.

Acuáticos: se proponen dos zonas principales para el desarrollo del hábitat más importante para la fauna del humedal; en uno de los sectores se establecerá una isla de junco de forma y perímetro irregular dentro del espejo de agua, la cual contribuirá a la diversidad de paisajes y ofrecerá áreas de refugio eficaz para la fauna.

Espejo de agua: El objetivo de esta zona es el restablecimiento de vegetación flotante y posibilitar el desarrollo de vegetación sumergida y semi sumergida, las cuales forman un hábitat óptimo para los macroinvertebrados que se encuentran en la cadena alimenticia de la avifauna.

Vegetación herbácea: áreas de esta importante vegetación, bien como representativa del humedal o bien por proveer hábitat esencial para fauna.

El uso principal de esta zona es atracción, mantenimiento y conservación de la biodiversidad. Aquí es compatible la contemplación y disfrute de la naturaleza y actividades no invasivas de recreación y educación. Con un uso condicionado a las situaciones particulares se encuentra la investigación, y entre los usos prohibidos están la recreación activa, cacería e ingreso de fauna. 


\section{ZONAS DE PRESERVAGIÓN Y PROTEGGIÓN AMBIENTAL}

Una vez esté establecida la vegetación acuática y terrestre, planteada como zona de protección para la fauna sin tránsito ni intervención alguna, excepto las labores necesarias de control de coberturas vegetales. Es indispensable destinar un sector exclusivamente para la conservación de los elementos de fauna y flora que sean factibles de restablecer en el área de los humedales.

\section{ZONA DE EDUGAGIÓN Y REGREAGIÓN PASIVA}

Se plantea una serie de senderos de integración para el tránsito, interpretación, educación y recreación pasiva de los visitantes. Se propone la construcción de un sendero perimetral que permita un tránsito que no sacrifique área de los humedales, permitiendo que bajo este pueda crecer vegetación acuática. Una infraestructura de paso que esté elevada del suelo permite una mejor visibilidad. Es importante emplear los materiales más apropiados para una construcción ecológicamente sostenible, que sea segura, que involucre temáticas de educación y control sobre la disposición de basura. También se propone la instalación de un puente conector de espacios de aprovechamiento del humedal, así como la instalación de observatorios sencillos, que sean la prolongación de los senderos.

Uso principal: recreación pasiva y contemplación de la naturaleza.

Usos compatibles: visitas educativas guiadas, actividades de interpretación y observatorios de investigación.

En otro orden de ideas, el enfoque ecosistémico representa para el manejo de los humedales urbanos la estrategia general para el manejo de la tierra, el agua, los recursos vivos y el mantenimiento y restauración de los sistemas naturales, sus funciones y sus valores, de tal manera que se promueva la conservación y el uso sostenible de una forma justa y equitativa mediante la integración de los factores ecológicos, económicos y sociales dentro de un marco geográfico definido principalmente por los límites.

Lo anterior implica reconocer en los programas y proyectos definidos en el plan la integración que existe entre la naturaleza y la cultura, siendo los seres humanos parte integrante de los ecosistemas y teniendo en cuenta las diferentes escalas de valoración, asumiendo como referente que se debe hacer un análisis de las funciones ambientales y los valores sociales de los humedales en las escalas de aproximación de paisaje, sistema y sitio. De acuerdo con esto, lo imprescindible es la educación ambiental no formal.

Por ende, la estrategia de investigación busca fomentar el conocimiento científico y técnico, así mismo potenciar el saber popular para retroalimentar la base de información en la toma de decisiones para el manejo de cada uno de los componentes físico, biótico y social en los humedales. La investigación, en la perspectiva del diálogo de saberes, pretende construir conocimiento social sobre el territorio que alberga el ecosistema y será uno de los pilares sobre los cuales se sustentará la recuperación de las condiciones físicas, ecológicas y socioculturales de cada humedal y su área de influencia.

La educación, comunicación y participación son también estrategias cuyo objetivo es la construcción colectiva de conocimiento sobre el humedal y su territorio, a fin de lograr procesos de apropiación social, fomento de actitudes proactivas por parte de la comunidad del área de influencia hacia los humedales, el uso y disfrute del ecosistema, garantizando la sustentabilidad de estos. En cuanto a la recuperación, protección, manejo y uso sostenible, son estrategias entendidas como la búsqueda de desarrollos armónicos entre las proyecciones de crecimiento poblacional, consolidación de la red urbana y la necesidad de recuperar los atributos, funciones y dinámicas de los ecosistemas del humedal, asegurando la calidad ambiental, la distribución equitativa de los beneficios ambientales, el bienestar de la sociedad y la búsqueda del paradigmático desarrollo sostenible.

De igual forma, la estrategia de coordinación o gestión interinstitucional se basa en el supuesto que para el logro de los objetivos la cooperación retribuye mayores beneficios que la competencia. Una correcta coordinación maximiza la eficiencia en la gestión ambiental, evitando la repetición de esfuerzos y fracasos, a la vez que genera un ambiente de cordialidad y confianza entre los actores.

De lo expuesto se desprende el plan de acción que está estructurado en programas como unidades lógicas de las acciones que deben rea- 
lizarse para el logro de los objetivos planteados por medio de la ejecución de los proyectos que los componen. La estructura de los programas y proyectos contenidos responden a las problemáticas identificadas a lo largo de la formulación participativa del modelo teórico.

Los programas y proyectos definidos en el manejo ambiental para la recuperación de los humedales urbanos son:

a. Programa: Saneamiento Predial. Proyecto: Adquisición predial para la recuperación integral de los humedales con el objetivo de garantizar las áreas necesarias para adelantar los proyectos enfocados a la recuperación y al disfrute de su oferta ambiental, social e hídrica.

b. Programa: Saneamiento Hídrico. Proyecto: Identificación y eliminación de las conexiones erradas en los colectores y cuencas aferentes con el objetivo de disminuir impactos ambientales generados por conexiones erradas en los humedales.

c. Programa: Recuperación Ecológica. Proyecto: Restauración de hábitats acuáticos y semiacuáticos de los humedales. Sub -Proyectos:

- Reconformación hidrogeomorfológica de los humedales y abastecimiento hídrico, con el objetivo de adecuar hidrogeomorfológicamente los cuerpos de agua, garantizando la sostenibilidad hídrica y biótica.

- Revegetalización acuática y control de plantas invasoras en el área inundable del humedal, cuyo objetivo es recuperar y aumentar la oferta y diversidad de la cobertura vegetal terrestre propia de los humedales, para su mantenimiento como parte de la estructura ecológica principal.
- Revegetalización terrestre y control de plantas invasoras en la zona de ronda del humedal, con el objetivo de recuperar y aumentar la oferta y diversidad de la cobertura vegetal terrestre propia de los humedales, para su mantenimiento como parte de la estructura ecológica.

d. Programa: Mecanismos de Manejo y Protección Socio Ambiental. Proyectos:

- Administración y manejo integral de los humedales, con el objetivo de garantizar la protección, conservación y sostenibilidad ecosistémica y de las obras e infraestructuras construidas en ellos.

- Cierre perimetral de los humedales, con el objetivo de controlar los factores de alteración sobre áreas de alta vulnerabilidad ecológica en los humedales.

- Diseño y construcción del área para administración e infraestructura para la educación ambiental.

Sub-Proyectos:

- Diseño y construcción del área para administración, cuyo objetivo es lograr que los humedales cuenten con la infraestructura física necesaria desde donde se puedan coordinar todas las actividades de mantenimiento, control, vigilancia, investigación y educación que se desarrollen en los mismos.

- Diseño y adecuación de senderos ecológicos y observatorios, con el objetivo de facilitar las labores de administración, vigilancia, mantenimiento y control, la investigación 
y la apropiación social de los humedales.

e. Programa: Gestión Social e Interinstitucional para la apropiación de los humedales como bienes públicos y patrimonio colectivo de la ciudad. Proyectos:

- Formación para el desarrollo de competencias ambientales ciudadanas, con el objetivo de generar competencias ambientales ciudadanas, mediante estrategias comunicativas y educativas que garanticen una gestión social del ecosistema de humedales para su conservación, así como la construcción de conocimiento social del territorio.

- Fortalecimiento de las organizaciones sociales y ambientales, con el objetivo de generar mecanismos que propicien el fortalecimiento de las organizaciones sociales y ambientales relacionadas con los humedales, así como su interacción con las entidades distritales, en pro de garantizar el cumplimiento de las acciones encaminadas a la recuperación y sostenibilidad ambiental de estos ecosistemas.

f. Programa: Investigación Aplicada y estudios técnicos. Proyectos:

- Estudio de aporte de agua subsuperficial: con el objetivo de identificar si existen posibles interacciones de los humedales con cuerpos de agua freáticos. Estudio de potencialidades de conectividad ecológica con su entorno urbano, con el objetivo de establecer las potencialidades de conectividad ecológica con las que contaría los humeda- les teniendo en cuenta su cuenca hidrográfica y la vegetación circundante.

- Relaciones vegetación fauna silvestre en los principales hábitats de los humedales, con el objetivo de determinar las relaciones existentes y potenciales entre los diferentes hábitats, las comunidades vegetales y las especies de fauna silvestre en los humedales.

g. Programa: Seguimiento y Monitoreo. Proyecto:

- Seguimiento a la recuperación ecológica, con el objetivo de conocer los cambios en los humedales en el tiempo y determinar el efecto de las actividades de restauración ecológica sobre los mismos.

De acuerdo con lo planteado se desprende una serie de acciones y tareas que conforman el plan de acción, referido a la gestión social e interinstitucional para la apropiación de los humedales como bien público y patrimonio colectivo de la ciudad, contentivo de una serie de actividades de educación ambiental, que se enfocan en la conservación y manejo de los ecosistemas urbanos, involucrando a las instituciones competentes.

\section{P R O G E S O \\ PAR T I C I PA T I VO E N LA \\ EJECUGIÓN DEL PLAN \\ D E MANEJOAMBIE N TAL \\ DE LOS H U E D A E S U R B A N O S}

En la ejecución del plan de manejo ambiental de los humedales el proceso participativo con la comunidad del área de influencia es de vital importancia en lo que se refiere a las charlas y talleres, con el fin de generar concienciación de uso de la tierra y el cuidado de los humedales, seleccionando los espacios como los patios de las casas, puesto que estos son utilizados para ubicar los desperdicios y chatarras y representan el frente del humedal. De tal ma- 
nera que se deben aprovechar estos espacios (patios) para la implementación de huertos e intercambiar los saberes y prácticas holísticas ancestrales de siembra.

Dentro de la línea estratégica "Agroecología", por ejemplo, se tiene el programa de acción social de agricultura urbana, y dentro de la línea estratégica "Intercambio sustentable", que no es más que el intercambio de saberes entre los pobladores, el cual despierta el interés de los presentes para el aprovechamiento de los espacios, patios y terrenos vacíos en los alrededores de los humedales, con el establecimiento de huertos de plantas medicinales y mejorar el ornato con plantas decorativas. Las estrategias educativas deben ir orientadas a la exposición previa sobre el tema, un díptico explicativo y el conversatorio. Este último se fundamenta en el intercambio de costumbres y tradiciones del uso de las plantas medicinales y prácticas holísticas referidas a la influencia de las fases lunares (la siembra en cuarto creciente y la poda en cuarto menguante), el día de la semana (el viernes como el mejor día para este tipo de actividades), y del mes más adecuado para la siembra y poda de las plantas; la cosecha de los frutales se debe realizar en tempranas horas del día, preferiblemente, para causar menos daño a las plantas, entre otros. El recorrido de campo es esencial como complemento de estas actividades.

\section{F I G U R A 11 。}

Plan de manejo en los humedales urbanos. Fuente: elaboración propia (2021).
Los resultados y logros deben ir hacia el establecimiento de modelos de siembra para el aprovechamiento y ornato de los espacios de los humedales, el enriquecimiento de la cultura popular, tradiciones y costumbres de la población y ejecución de otras actividades como la arborización. Entre los acuerdos están el compromiso de los participantes de tener buenas disposiciones de selección de nuevos terrenos a plantar con especies que así se requieran y lo más importante, el compromiso de cuidar y vigilar los espacios de los humedales.

Por último, es bien importante la evaluación de las actividades valorando la participación educativa conservacionista para concienciar y actuar como efecto multiplicador en la comunidad, el conocimiento de la existencia de los humedales urbanos como espacios recreativos, conocimiento de la funciones de los humedales, continuación del cumplimiento de actividades de conservación, contribución de las jornadas al mejoramiento de conducta de la comunidad a la protección del ambiente, la participación con agrado de la comunidad en las jornadas y la incorporación de otros miembros de la comunidad a la conservación de los espacios. Se determina, entonces, una interrelación conjunta con todos los aspectos aquí considerados, como se puede ver en la siguiente figura.

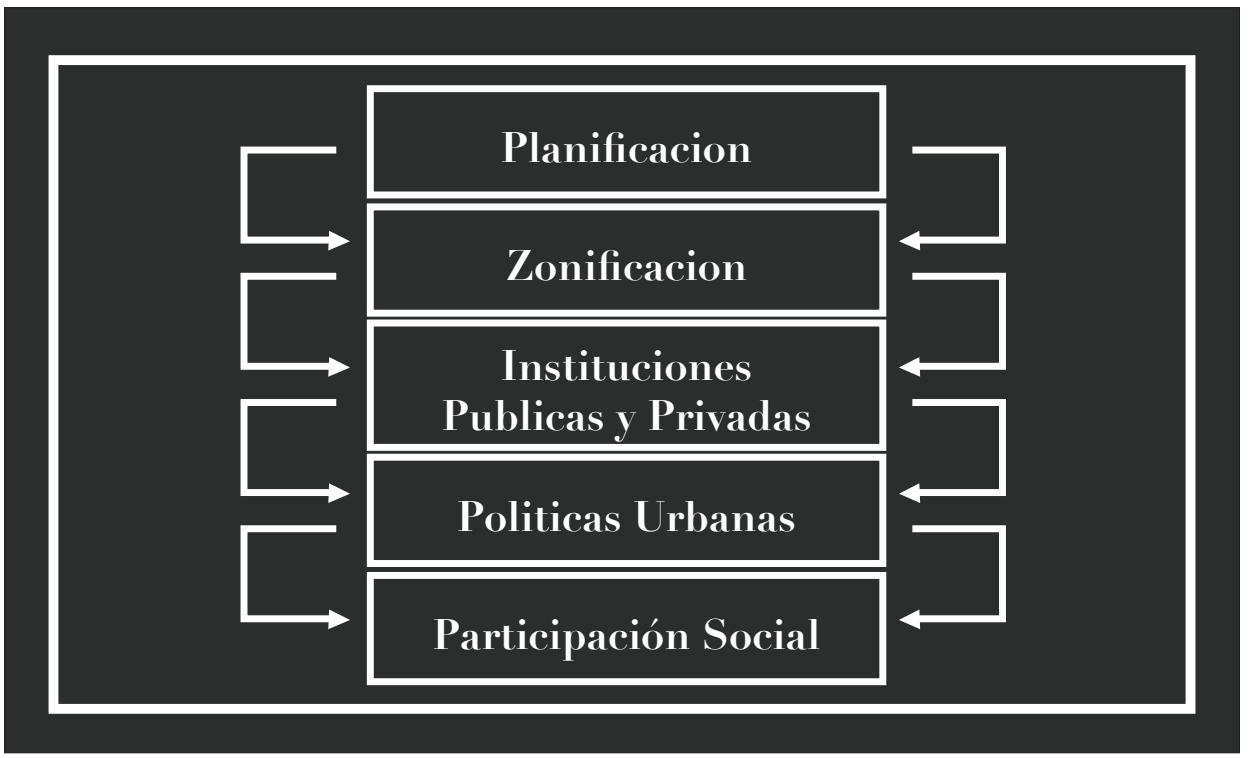

